\title{
Late Time Behavior of Unstable Particles
}

\author{
K. Urbanowski", J. Piskorski \\ University of Zielona Gora, Institute of Physics, ul. Prof. Z. Szafrana 4a, 65-516 Zielona Gora, Poland \\ K.Urbanowski@proton.if.uz.zgora.pl, jaropis@proton.if.uz.zgora.pl
}

\begin{abstract}
Possible consequences of non-exponential behavior of the survival amplitude of an unstable particle at very late times are considered. We describe a new quantum phenomenon: Analyzing the transition time region between exponential and post-exponential form of the survival amplitude we find that the instantaneous energy of the considered unstable state can take large values, much larger than the energy of this state for $t$ from the exponential time region. We find also that the instantaneous energy of the unstable state tends to the minimal energy of the system $E_{\min }$ as $t \rightarrow \infty$ which is much smaller than the energy of this state for $t$ of the order of the lifetime of the considered state. Taking into account the results obtained for the model considered, it is hypothesized that this purely quantum mechanical effect may be responsible for the properties of broad resonances such as $\sigma$ meson as well as having astrophysical consequences.
\end{abstract}

Keywords Non-Exponential Decay, Unstable States

\section{Introduction}

Almost all known elementary particles are unstable. So, the knowledge about the properties of unstable particles is one of the fundamental problems in physics. The behavior of unstable states $|\phi\rangle \in H$ (where $H$ is the Hilbert space of states of the considered system) is characterized by their decay law, which is defined by means of a survival probability amplitude, $a(t)$, of finding the system at the time $t$ in the initial state $|\phi\rangle$ prepared at time $t_{0}=0$,

$$
\begin{aligned}
a(t) & =\langle\phi \mid \phi(t)\rangle . \\
i \hbar \frac{\partial}{\partial t}|\phi(t)\rangle & =\mathrm{H}|\phi(t)\rangle,
\end{aligned}
$$

where $\mathrm{H}$ denotes the total selfadjoint Hamiltonian for the system considered.

Using the survival amplitude $a(t)$ one defines the decay law, $P_{\phi}(t)$, of an unstable state $|\phi\rangle$ decaying in vacuum as follows

$$
\begin{gathered}
P_{\phi}(t)=|a(t)|^{2}, \\
a(t)=\int_{\text {Spec. (H) }} \omega(E) e^{-\frac{i}{\hbar} E t} d E .
\end{gathered}
$$

The survival amplitude $a(t)$, and thus the decay law $P_{\varphi}(t)$ of the unstable state $|\varphi\rangle$, can be written as the Fourier transform of the density of the energy distribution $\omega(E)$ for the system in this state[1,2] and $|\varphi(t)\rangle$ is the solution of the

* Corresponding author:

K.Urbanowski@proton.if.uz.zgora.pl (K. Urbanowski)

Published online at http://journal.sapub.org/jnpp

Copyright (C) 2012 Scientific \& Academic Publishing. All Rights Reserved
Schrödinger equation for the initial condition $|\varphi(0)\rangle=|\varphi\rangle$ : where $\omega(E) \geq 0$ and $a(0)=1$.

Khalfin[3] assuming that the spectrum of $\mathrm{H}$ must be bounded from below, $(\operatorname{Spec} .(\mathrm{H})>-\infty)$, and using the Paley-Wiener Theorem[4] proved that in the case of unstable states there must be

$$
|a(t)| \geq A \exp \left[-b t^{q}\right] \text { for }|t| \rightarrow \infty,
$$

(where $A>0 b>0$ and $0<q<1$ ).

In an experiment described in the Rothe paper[5], the experimental evidence of deviations from the exponential decay law at long times was reported. This result gives rise to another problem which now becomes important: If (and how) deviations from the exponential decay law at long times affect the energy of the unstable state and its decay rate at this time region.

Note that in fact the amplitude $a(t)$ contains information about the decay law $P_{\varphi}(t)$ of the state $|\varphi\rangle$, that is about the decay rate $\Gamma_{\varphi}^{0} \equiv \frac{\hbar}{\tau_{\varphi}},\left(\tau_{\phi}\right.$ is the lifetime $)$, of this state, as well as the energy $E_{\varphi}^{0}$ of the system in this state. This information can be extracted from $a(t)$, e. g., using the "effective Hamiltonian" $h_{\varphi}$ (for details see[6, 7]). In the considered case this $h_{\varphi}$ acts in the one-dimensional subspace of states $H_{\|}$spanned by the normalized vector $|\varphi\rangle$ and it is defined as follows (see, eg.[6]),

$$
h_{\phi} \stackrel{\mathrm{d} e f}{=} i \hbar \frac{\partial a(t)}{\partial t} \frac{1}{a(t)} \text {. }
$$

In general, $h_{\varphi}$ can depend on time $t, h_{\varphi} \equiv h_{\varphi}(t)$. Within the one pole approximation which is valid to a good approximation for times $t \propto \tau_{\varphi}$, we have 


$$
\left.a(t) \approx a_{0}(t)=\operatorname{ex} \mathbb{p}^{-} \frac{i}{\hbar}\left(E_{\varphi}^{0}-\frac{i}{2} \Gamma_{\varphi}^{0}\right) t\right],
$$

where $E_{\varphi}^{0}, \frac{1}{2} \Gamma_{\varphi}^{0}$ are the pole coordinates, and simply

$$
h_{\phi} \approx h_{\phi}^{0} \equiv E_{\phi}^{0}-\frac{i}{2} \Gamma_{\phi}^{0} .
$$

It is easy to show that equivalently[7]

$$
h_{\phi}(t) \equiv \frac{\langle\phi|\mathrm{H}| \phi(t)\rangle}{\langle\phi \mid \phi(t)\rangle} .
$$

One meets effective Hamiltonians of this type when one starts with the time-dependent Schrödinger equation (2) for the total state space $H$ and looks for the rigorous evolution equation for the distinguished subspace of states $H_{\|} \subset H$ (see[6] and references one finds therein). In the case of one-dimensional $H_{\|}$this rigorous Schrödinger-like evolution equation has the following form for the initial condition $a(0)=1,[6]$,

$$
i \hbar \frac{\partial a(t)}{\partial t}=h_{\phi}(t) a(t)
$$

Relations (6) and (10) establish a direct connection between the amplitude $a(t)$ for the state $|\phi\rangle$ and the exact effective Hamiltonian $h_{\phi}(t)$ governing the time evolution in the one-dimensional subspace $H_{\|} \ni|\phi\rangle$. Thus, the use of the evolution equation (10) or the relation (6) is one of the most effective tools for the accurate analysis of the early- as well as the long-time properties of the energy and decay rate of a given quasi-stationary state $|\phi(t)\rangle$.

So let us assume that we know the amplitude $a(t)$. Then starting with this $a(t)$ and using the expression (6) one can calculate the effective Hamiltonian $h_{\phi}(t)$ in a general case for every $t$. Thus, one finds the following expressions for the instantaneous energy and the instantaneous decay rate of the system in the state $|\phi\rangle$ under considerations, (for details see:[7]),

$$
\begin{array}{r}
E_{\phi}(t)=\mathfrak{R}\left(h_{\phi}(t),\right. \\
\Gamma_{\phi}(t)=-2 \mathfrak{I}\left(h_{\phi}(t),\right.
\end{array}
$$

where $\mathfrak{R}(z)$ and $\mathfrak{I}(z)$ denote the real and imaginary parts of $z$ respectively.

Asymptotic long time properties of the instantaneous energy $E_{\phi}(t)$ were analyzed in our previous papers for a model of the type considered in Sec. 2 (see[7]) and in a general case[8]). The aim of this paper is to analyze the long time properties of $E_{\phi}(t)$ for the transition times region between the exponential and non-exponential form of the survival amplitude using $a(t)$ calculated for the given density $\omega(E)$ and to discuss possible consequences of these properties.

\section{The model}

Let us assume that $\operatorname{Spec} .(\mathrm{H})=\left[E_{\min }, \infty\right)$, (where, $\left.E_{\text {min }}>-\infty\right)$, and let us choose $\omega(E)$ as follows (compare[9])

$$
\begin{gathered}
\omega(E)=\frac{N}{2 \pi} \Theta\left(E-E_{\text {min }}\right) \frac{\Gamma_{\phi}^{0}}{\left(E-E_{\phi}^{0}\right)^{2}+\left(\frac{\Gamma_{\phi}^{0}}{2}\right)^{2}} \\
\equiv \omega_{B W}\left(E, E_{\text {min }}\right),
\end{gathered}
$$

where $N$ is a normalization constant chosen so that $a(0)=1$ and $\Theta(E)=\{1$ for $E \geq 0$ and 0 for $E<0\}$. For such $\omega_{B W}(E)$ using (4) one finds

$$
\begin{gathered}
a(t)=N e^{-\frac{i}{\hbar} h_{\phi}^{0} t}\left\{1-\frac{i}{2 \pi} \times\right. \\
\times\left[e^{\frac{\Gamma_{\phi}^{0}}{\hbar}} E_{1}\left(-\frac{i}{\hbar}\left(h_{\phi}^{0}-E_{\text {min }}+i \Gamma_{\phi}^{0}\right) t\right)\right. \\
\left.\left.-E_{1}\left(-\frac{i}{\hbar}\left(h_{\phi}^{0}-E_{\text {min }}\right) t\right)\right]\right\},
\end{gathered}
$$

where $E_{1}(x)$ denotes the integral--exponential function[9, $10]$.

In general one has

$$
a(t) \equiv a_{\exp }(t)+a_{n o n}(t)
$$

where

$$
a_{\text {exp }}(t)=N e^{-\frac{i}{\hbar} h_{\varphi}^{0} t}, \quad a_{\text {non }}(t)=a(t)-a_{\text {exp }}(t) .
$$

Making use of the asymptotic expansion of $E_{1}(x)$ [10]

$$
\left.E_{1}(z)\right|_{|z| \rightarrow \infty} \propto \frac{e^{-z}}{z}\left(1-\frac{1}{z}+\frac{2}{z^{2}}-\ldots\right),
$$

where $|\arg z|<\frac{3}{2} \pi$, one concludes that

$$
\begin{gathered}
\left.a(t)\right|_{t \rightarrow \infty} \approx N e^{-\frac{i}{\hbar} h_{\phi}^{0} t} \\
+\frac{N}{2 \pi} e^{-\frac{i}{\hbar} E_{\text {min }}^{t}}\left\{(-i) \frac{\Gamma_{\phi}^{0}}{\left|h_{\phi}^{0}-E_{\text {min }}\right|^{2}} \frac{\hbar}{t}\right. \\
\left.-2 \frac{\left(E_{\phi}^{0}-E_{\text {min }}\right) \Gamma_{\phi}^{0}}{\left|h_{\phi}^{0}-E_{\text {min }}\right|^{4}}\left(\frac{\hbar}{t}\right)^{2}+\ldots\right\}
\end{gathered}
$$

and

$$
\begin{aligned}
\left.h_{\phi}(t)\right|_{t \rightarrow \infty} & =\left.i \hbar \frac{\partial a(t)}{\partial t} \frac{1}{a(t)}\right|_{t \rightarrow \infty} \\
& \approx E_{\text {min }}-i \frac{\hbar}{t}
\end{aligned}
$$




$$
-2 \frac{E_{\phi}^{0}-E_{\min }}{\left|h_{\phi}^{0}-E_{\min }\right|^{2}}\left(\frac{\hbar}{t}\right)^{2}+\ldots
$$

for the considered case (13) of $\omega_{B W}(E)$ (for details see[7]). From (18) it follows that

$$
\begin{gathered}
\mathfrak{R}\left(\left.h_{\phi}(t)\right|_{t \rightarrow \infty}\right) \stackrel{\mathrm{d} e f}{=} E_{\phi}^{\infty}(t) \\
\approx E_{\text {min }}-2 \frac{E_{\phi}^{0}-E_{\text {min }}}{\left|h_{\phi}^{0}-E_{\text {min }}\right|^{2}}\left(\frac{\hbar}{t}\right)^{2} \\
\rightarrow E_{\text {min }}, \\
t \rightarrow \infty
\end{gathered}
$$

where $E_{\varphi}^{\infty}(t)=\left.E_{\varphi}(t)\right|_{t \rightarrow \infty}$, and

$$
\mathfrak{I}\left(\left.h_{\phi}(t)\right|_{t \rightarrow \infty}\right) \approx-\frac{\hbar}{t} \underset{t \rightarrow \infty}{\rightarrow} 0 .
$$

The property (19) means that

$$
\mathfrak{R}\left(\left.h_{\phi}(t)\right|_{t \rightarrow \infty}\right) \equiv E_{\phi}^{\infty}(t)<E_{\phi}^{0} .
$$

Methods used in asymptotic analysis allow one to find a form of (4) for large $t$ for all densities $\omega(E)$ for which the Fourier transform (4) exists. The same can be done for the derivative of $a(t)$ and then using (6) or (9) a general asymptotic form of $h_{\phi}(t)$ can be found. It looks as follows

$$
\left.h_{\varphi}(t)\right|_{t \rightarrow \infty} \approx E_{\min }+\left(-\frac{i \hbar}{t}\right) c_{1}+\left(-\frac{i \hbar}{t}\right)^{2} c_{2}+\ldots,
$$

where $c_{i}=c_{i}^{*}, i=1,2, \ldots$ (compare[8]).

Note that from (17) one obtains

$$
\begin{aligned}
& \left.|a(t)|_{t \rightarrow \infty}\right|^{2} \approx N^{2} e^{-\frac{\Gamma_{\phi}^{0}}{\hbar} t} \\
& +\frac{N^{2}}{\pi} \sin \left[\left(E_{\phi}^{0}-E_{\min }\right) t\right] \times \\
& \quad \times e^{-\frac{1}{2} \frac{\Gamma_{\phi}^{0}}{\hbar} t} \frac{\Gamma_{\phi}^{0}}{\left|h_{\phi}^{0}-E_{\min }\right|^{2}} \frac{\hbar}{t} \\
& +\frac{N^{2}}{4 \pi^{2}} \frac{\left(\Gamma_{\phi}^{0}\right)^{2}}{\left|h_{\phi}^{0}-E_{\min }\right|^{4}} \frac{\hbar^{2}}{t^{2}}+\ldots .
\end{aligned}
$$

Relations (17) - (21) become important for times $t>t_{a s}$, where $t_{a s}$ denotes the time $t$ at which contributions to $\left.|a(t)|_{t \rightarrow \infty}\right|^{2}$ from the first exponential component in (23) and from the third component proportional to $1 / t^{2}$ are comparable. So $t_{a s}$ can be be found by considering the following relation

$$
e^{-\frac{\Gamma_{\phi}^{0}}{\hbar} t} \approx \frac{1}{4 \pi^{2}} \frac{\left(\Gamma_{\phi}^{0}\right)^{2}}{\left|h_{\phi}^{0}-E_{\min }\right|^{4}} \frac{\hbar^{2}}{t^{2}} .
$$

Assuming that the right hand side is equal to the left hand side in the above relation one gets a transcendental equation. Exact solutions of such an equation can be expressed by means of the Lambert $W$ function[11]. An asymptotic solution of the equation obtained from relation (24) is relatively easy to find[12]. The very approximate asymptotic solution, $t_{a s}$, of this equation for $\left(\frac{E_{\phi}}{\Gamma_{\phi}^{0}}\right)>10$ (in general for $\left.\left(\frac{E_{\phi}}{\Gamma_{\phi}^{0}}\right) \rightarrow \infty\right)$ has the form

$$
\begin{gathered}
\frac{\Gamma_{\phi}^{0} t_{a s}}{\hbar} \approx 8,28+4 \ln \left(\frac{E_{\phi}^{0}-E_{\min }}{\Gamma_{\phi}^{0}}\right) \\
+2 \ln \left[8,28+4 \ln \left(\frac{E_{\phi}^{0}-E_{\min }}{\Gamma_{\phi}^{0}}\right)\right]+\ldots
\end{gathered}
$$

\section{Numerical Calculations}

Long time properties of the survival probability $|a(t)|^{2}$ and instantaneous energy $E_{\phi}(t)$ are relatively easy to find analytically for times $t>>t_{a s}$ even in the general case as it was shown in[8]. It is much more difficult to analyze these properties analytically in the transition time region where $t \approx t_{a s}$. It can be done numerically for some models (see[13]).

The model considered in Sec. 2 and defined by the density $\omega_{B W}(E),(13)$, allows one to find numerically the decay curves and the instantaneous energy $E_{\phi}(t)$ as a function of time $t$. The results presented in this Section have been obtained assuming for simplicity that the minimal energy $E_{\min }$ appearing in the formula (13) is equal to zero, $E_{\min }=0$. So, all numerical calculations were performed for the density $\widetilde{\omega}_{B W}(E)$ given by the following formula

$$
\begin{aligned}
\widetilde{\omega}_{B W}(E)= & \frac{N}{2 \pi} \Theta(E) \frac{\Gamma_{\phi}^{0}}{\left(E-E_{\phi}^{0}\right)^{2}+\left(\frac{\Gamma_{\phi}^{0}}{2}\right)^{2}} \\
& \equiv \omega_{B W}\left(E, E_{\text {min }}=0\right),
\end{aligned}
$$

for some chosen $\frac{E_{\phi}^{0}}{\Gamma_{\phi}^{0}}$. Performing calculations particular attention was paid to the form of the probability $|a(t)|^{2}$, i. e. of the decay curve, and of the instantaneous energy $E_{\phi}(t)$ for times $t$ belonging to the most interesting transition time-region between exponential and non-exponential parts 
of $|a(t)|^{2}$, where the following relation corresponding with (24) takes place,

$$
\left|a_{\text {exp }}(t)\right|^{2} \approx\left|a_{\text {non }}(t)\right|^{2},
$$

where $a_{e x p}(t), a_{\text {non }}(t)$ are defined by (15). Results are presented graphically below in Figs (1) - (6).

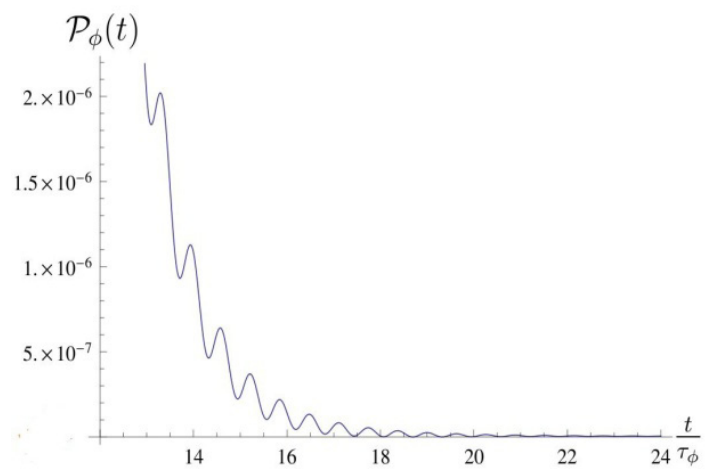

Figure 1. Survival probability $P_{\phi}(t)=|a(t)|^{2}$ in the transition time region. The case $\frac{E_{\phi}^{0}}{\Gamma_{\phi}^{0}}=10$.

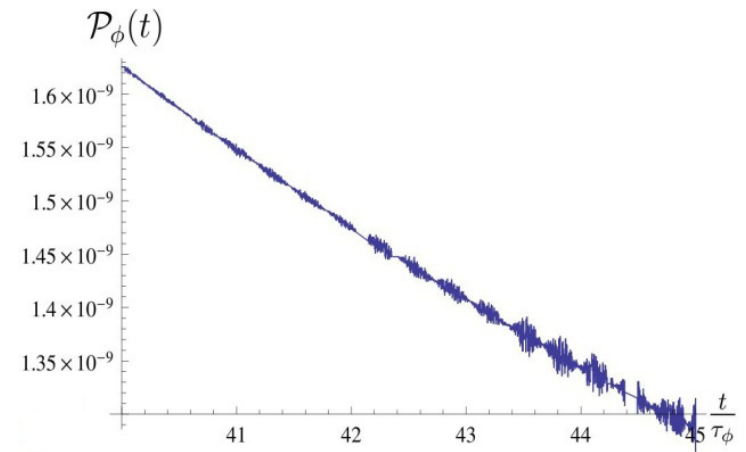

Figure 2. Survival probability $P_{\phi}(t)=|a(t)|^{2}$ in the transition time region. The case $\frac{E_{\phi}^{0}}{\Gamma_{\phi}^{0}}=10$ for times $t$ longer than those in Fig. 1.

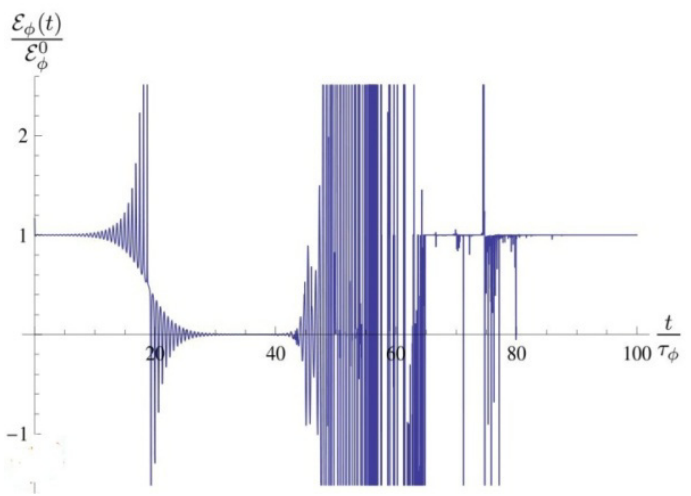

Figure 3. Instantaneous energy $E_{\phi}(t)$ in the transition time region. The case $\frac{E_{\phi}^{0}}{\Gamma_{\phi}^{0}}=10$.

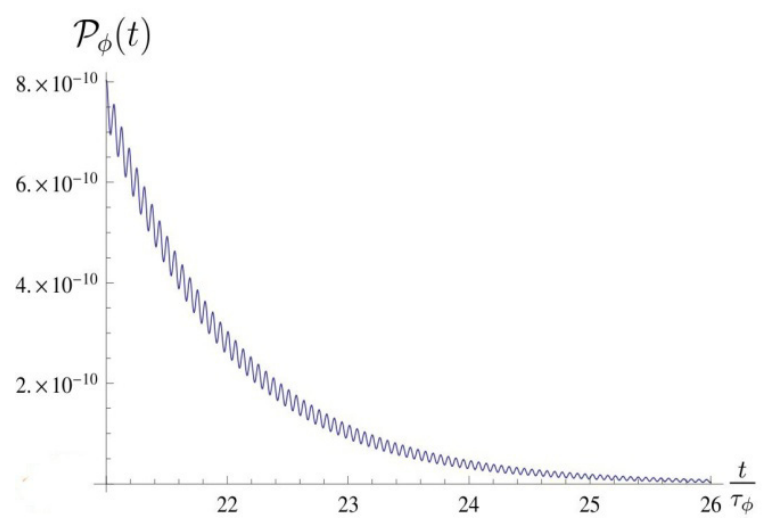

Figure 4. Survival probability $P_{\phi}(t)=|a(t)|^{2}$ in the transition time region. The case $\frac{E_{\phi}^{0}}{\Gamma_{\phi}^{0}}=100$.

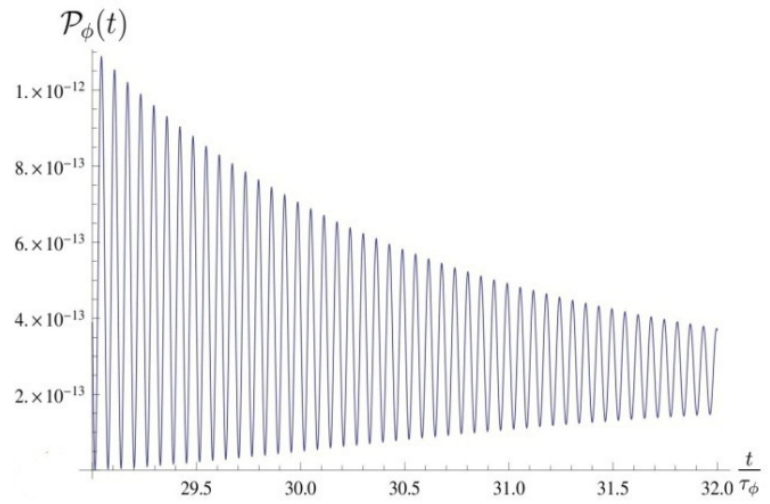

Figure 5. Survival probability $P_{\phi}(t)=|a(t)|^{2}$ in the transition time region. The case $\frac{E_{\phi}^{0}}{\Gamma_{\phi}^{0}}=100$ for times $t$ longer than those in Fig. 4.

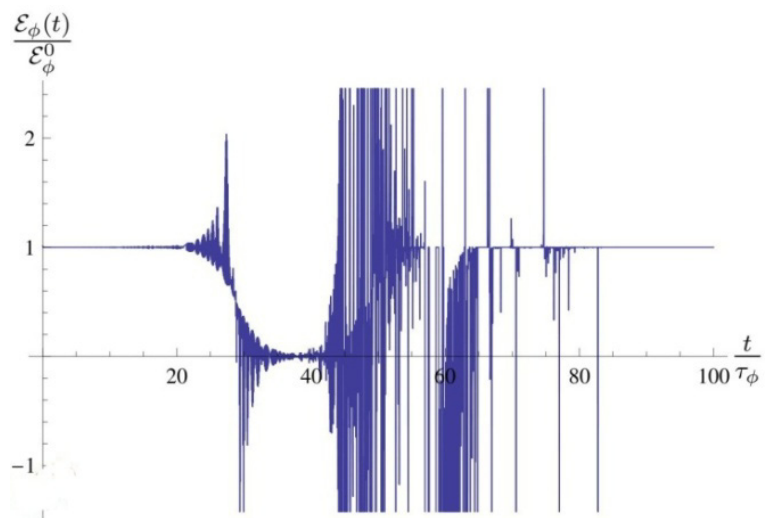

Figure 6. Instantaneous energy $E_{\phi}(t)$ in the transition time region. The case $\frac{E_{\phi}^{0}}{\Gamma_{\phi}^{0}}=100$

\section{Discussion: Possible Hyphotetical Effects}


Decay curves of type shown in Fig. (1), Fig. (2), Fig. (4), Fig. (5) can be encountered for a very large class of models defined by energy densities $\omega(E)$ of the following type $($ see[2, 14]),

$$
\begin{gathered}
\omega(E)=\frac{N}{2 \pi} \Theta\left(E-E_{\text {min }}\right)\left(E-E_{\text {min }}\right)^{\lambda} \times \\
\times \frac{\Gamma_{\phi}^{0}}{\left(E-E_{\phi}^{0}\right)^{2}+\frac{\left(\Gamma_{\phi}^{0}\right)^{2}}{4}} f(E) \\
\equiv \omega_{B W}\left(E, E_{\text {min }}\right)\left(E-E_{\text {min }}\right)^{\lambda} f(E),
\end{gathered}
$$

where $\lambda \geq 0, f(E)$ is a form-factor - it is a smooth function going to zero as $E \rightarrow \infty$ and it has no threshold and no pole. The asymptotical large time behavior of $a(t)$ is due to the term $\left(E-E_{\text {min }}\right)^{\lambda}$ and the choice of $\lambda$ (see, eg.[2,8]). The Breit-Wigner part, $\omega_{B W}\left(E, E_{\text {min }}\right)$, of the density (28) is responsible for a behavior of $a(t)$ at exponential decay time region and at transition time region. The density $\omega(E)$ defined by the relation (28) fulfills all physical requirements and it leads to the decay curves of a very similar form at transition times region to the decay curves presented above. The characteristic feature of all these decay curves is the presence of sharp and frequent oscillations at the transition times region (see Figs (1), (2), (4), (5)) (see also, eg.[9],[15] -[20]). So derivatives of the amplitude $a(t)$ may reach extremely large values for some times from the transition time region and the modulus of these derivatives is much larger than the modulus of $a(t)$, which is very small for these times. This explains why in this time region the real and imaginary parts of $h_{\varphi}(t) \equiv E_{\varphi}(t)-\frac{i}{2} \Gamma_{\varphi}(t)$, which can be expressed by the relation (6), ie. by a large derivative of $a(t)$ divided by a very small $a(t)$, reach values much larger than the energy $E_{\varphi}^{0}$ of the unstable state measured at times for which the decay curve has the exponential form. For the model considered we found that, eg. for $\frac{E_{\varphi}^{0}}{\Gamma_{\varphi}^{0}}=10$ and $5 \tau_{\varphi} \leq t \leq 60 \tau_{\varphi}$ the maximal value of the instantaneous energy equals $E_{\varphi}(t)=89,2209 E_{\varphi}^{0}$ and $E_{\varphi}(t)$ reaches this value for $t \equiv t_{m x, 10}=53,94 \tau_{\varphi}$ and then the survival probability $P_{\varphi}(t)$ is of order $P_{\varphi}\left(t_{m x, 10}\right) \propto 10^{-9}$.

The question is whether and where this effect can manifest itself. It seems that the following two possibilities of observing the above long time properties of unstable states are worth considering: The first one is that one should analyze the properties of unstable states whose $t_{a s}$ values are not too big. The second one is finding a possibility to observe a suitably large number of events, i.e. unstable particles, created by the same source and living suitably long.

The problem with understanding the properties of broad resonances in the scalar sector ( $\sigma$ meson problem[21]) discussed in[18,22] and recently in[23] where the hypothesis was formulated that this problem could be connected with the properties of the decay amplitude in the transition time region, seems to be possible manifestations of this effect and this problem refers to the first possibility mentioned above. There is the problem with determining the mass of broad resonances. The measured range of possible mass of the $\sigma$ meson is very wide, $400-1200 \mathrm{MeV}$. So one can not exclude the possibility that the masses of some $\sigma$ mesons are measured for times of the order of their lifetime and some of them for times where their instantaneous energy $E_{\sigma}(t)$ is much larger. This is exactly the case presented in Fig. (3) and Fig. (6). For broad mesons the ratio $\frac{E_{\sigma}^{0}}{\Gamma_{\sigma}^{0}}$ is relatively small and thus the time $t_{a s}$, when the above discussed effect occurs, appears to be not too long.

Astrophysical and cosmological processes in which extremely huge numbers of unstable particles are created seem to be another possibility for the above discussed effect to become manifest. Rapid fluctuations of the instantaneous energy $E_{\varphi}(t)$ (see Figs 3 and 6) of unstable particles $\phi$ take place at times $t$ of order $t_{a s}$. Fluctuations of $E_{\varphi}(t)$ lead to fluctuations of instantaneous masses, $m_{\varphi}^{0}(t)$, of particles $\phi$,

$$
m_{\phi}^{0}(t)=\frac{1}{c^{2}} E_{\phi}(t) .
$$

Note now that results presented in Figures 3 and 6 were obtained for particles in their rest coordinate system. Unstable particles created by cosmic sources move extremely fast with a velocity $v^{\phi}(t)$ close to the speed of light $c$. The energy $W^{\phi}$ of such moving particles,

$$
W^{\phi}=m_{\phi}^{0}(t) c^{2} \gamma(t) \equiv E_{\phi}(t) \gamma(t),
$$

where $\gamma(t)=\left(1-\left(\frac{v^{\varphi}(t)}{c}\right)^{2}\right)^{-1 / 2}$, should be the same at times $t \triangleright \tau_{\phi}$ and at times $t \propto t_{a s}$. This follows from the principle of the energy conservation. From relation (30) one can infer that this is possible only when changes of $E_{\phi}(t)$ at times $t \propto t_{a s}$ are balanced with suitable changes of $\gamma(t)$ (i.e. of the velocity $\left.v^{\phi}(t)\right)$. So, in the case of moving unstable particles, rapid fluctuations (changes) of their velocities should be observed at distances $d^{\phi} \propto v^{\phi} t_{a s}$ from their source. It seems to be obvious that astrophysical and cosmological processes are the only sources able to create suitably large number of unstable particles guaranteeing that a part of them survives up to times $t \propto t_{a s}$. Therefore an analysis of these processes and observational data connected with them seems to be only chance to find possible effects caused by rapid fluctuations of velocities of unstable 
particles at late times.

It appears that properties of unstable states at times $t$ much later than $t_{\text {as }}$ described by the decay law (23) and relations (18), (19) and (22) may be useful when one tries to explain some cosmological processes. The late time behavior of false vacuum decay is an example of such processes[24, $25,26]$. The false vacuum[24,25] is metastable and space areas with false vacuum decay by tunneling to the space area with the true vacuum state. Some space areas with false vacuum can survive up o times $t>t_{a s}$ and then properties of these false vacuum states are described by relations (18), (19) and (22)[26]. At times $t \propto t_{\text {as }}$ the energy of the false vacuum state should fluctuate very fast according to the results presented in Fig. 3 na 6. This may also affect properties and a behavior of space bubbles with false vacua.

Note that all possible effects discussed in this paper are the simple consequence of the fact that the instantaneous energy $E_{\phi}(t)$ of unstable particles becomes large for suitably long times compared with $E_{\phi}^{0}$ and for some times even extremely large. This property of $E_{\phi}(t)$ is a purely quantum effect resulting from the assumption that the energy spectrum is bounded from below and it was found by performing a rigorous analysis of the properties of the quantum mechanical survival probability $a(t)$.

\section{REFERENCES}

[1] S. Krylov, V. A. Fock, Zh. Eksp. Teor. Fiz. 17, (1947), 93.

[2] L. Fonda, G. C. Ghirardii and A. Rimini, Rep. on Prog. in Phys. 41, (1978), 587.

[3] L. A. Khalfin, Zh. Eksp. Teor. Fiz. 33, (1957), 1371[Sov. Phys. - JETP] 6, (1958), 1053].

[4] R. E. A. C. Paley, N. Wiener, Fourier transforms in the complex domain, American Mathematical Society, New York, 1934.

[5] C. Rothe, S. I. Hintschich and A. P. Monkman, Phys. Rev. Lett. 96, (2006), 163601.

[6] K. Urbanowski, Phys. Rev. A 50, (1994), 2847.
[7] K. Urbanowski, Cent. Eur. J. Phys. 7, (2009), 696; DOI: 10.2478/s11534-009-0053-5.

[8] K. Urbanowski, Eur. Phys. J. D 54, (2009), 25.

[9] K. M. Sluis, E. A. Gislason, Phys. Rev. A 43, (1991), 4581.

[10] Handbook of Mathematical Functions, Natl. Bur. Stand. Appl. Math. Ser. No 55, eds. M. Abramowitz nad I. A. Stegun (U.S. GPO, Washington, D.C., 1964).

[11] R. M. Corless, G. H. Gonet, D. E. G. Hare, D. J. Jeffrey and D. E. Khnut, Adv. Comput. Math. 5, (1996), 329.

[12] F. W. J. Olver, Asymptotics and special functions, Academic Press, New York, 1974. W. Heitler, The Quantum Theory of Radiation, Oxford University Press, London 1954 and Dover Publications, New York 1984.

[13] K. Urbanowski, J. Piskorski, Energy of unstable states at long times, arXiv: 0908.2219.

[14] N. G. Kelkar, M. Nowakowski, K. P. Khemchadani, Phys. Rev. C 70, (2004), 024601.

[15] J. Bogdanowicz, M. Pindor and R. R a czka, Found. Phys. 25, (1995), 833.

[16] R. E. Parrot, J. Lawrence, Europhys. Lett. 57, (2002), 632.

[17] J. Lawrence, Journ. Opt. B: Quant. Semiclass. Opt. 4, (2002), S446.

[18] M. Nowakowski, N. G. Kelkar, AIP Conf. Proc. 1030, (2008), 250 - 255; arXiv: 0807.5103.

[19] G. Garcia-Calderon, J. L. Mateos and M. Moshinsky, Phys. Rev. Lett., 74, (1995), 337.

[20] G. Garcia-Calderon, I. Maldonado and J. Villavicencio, Phys. Rev. A 76, (2007), 012103.

[21] C. Amsler at al, Phys. Lett. B 667, (2008), 1.

[22] M. Nowakowski, N. G. Kelkar, Nishiharima 2004, Penataquark - Proceedings of International Workshop on PENATAQUARK 04, Spring - 8, Hyogo, Japan, 23 -24 July 2004, pp. 182 - 189; arXiv: hep-ph/0411317.

[23] N. G. Kelkar, M. Nowakowski, J. Phys. A: Math. Teor., 43, 385308 (2010).

[24] C. G. Callan, S. Coleman, Phys. Rev. D, 1 6, 1762 (1977); S. Coleman, F. De Lucia, Phys. Rev. D 21,3305 (1980).

[25] L. M. Krauss, J. Dent, Phys. Rev. Lett., 1 00, 171301 (2008).

[26] K. Urbanowski, Phys. Rev. Lett., 107, 209001 (2011). 